\title{
Avaliação da atividade antiulcerogênica da Maytenus truncata Reiss (Celastraceae)
}

\author{
J.L. Silva ${ }^{1}$, R.P. Silva', R.M. Jorge ${ }^{1}$, G.D. Fátima Silva ${ }^{2}$, S.A. Vieira Filho ${ }^{2}$, A.P.N.D. Fonseca ${ }^{2}$, \\ C.A. Tagliati $^{1 *}$
}

${ }^{1}$ Laboratório de Toxicologia Experimental, Departamento de Análises Clínicas e Toxicológicas, Faculdade de Farmácia, Universidade Federal de Minas Gerais, Av. Antônio Carlos 6627, Pampulha, 31270-010, Belo Horizonte, MG, Brasil,

${ }^{2} N E P L A M$, Departamento de Química, ICEx, Universidade Federal de Minas Gerais, Av. Antônio Carlos 6627, Pampulha, 31270-010, Belo Horizonte, MG, Brasil

\begin{abstract}
RESUMO: Maytenus truncata Reiss (Celastraceae) é uma planta nativa da Bahia (Brasil), sendo conhecida como "espinheira-santa". É usada popularmente na forma de decoto das folhas (chás) como antiulcerogênico, similarmente à Maytenus ilicifolia, a verdadeira "espinheira-santa". O objetivo do presente estudo foi avaliar a atividade antiúlcera e cicatrizante, assim como o perfil fitoquímico dos extratos brutos em acetato de etila e metanol da Maytenus truncata. A administração per os desses extratos nas doses de $120 \mathrm{mg} / \mathrm{kg}$ e $240 \mathrm{mg} / \mathrm{kg}$ reduziu a severidade da lesão gástrica induzida pelo estresse ao frio $\left(-18{ }^{\circ} \mathrm{C}\right.$ por 45 minutos $)$ em ratos, com resultados mais significativos para o extrato bruto obtido em metanol. A administração dos extratos provocou o aumento do pH. Os resultados obtidos na administração do extrato bruto em metanol não contrariam seu uso popular, não somente pela atividade observada, mas também por se tratar de um extrato de alta polaridade cujos princípios podem ser obtidos a partir de uma infusão, embora estudos clínicos devam ser realizados para confirmação dessa hipótese.
\end{abstract}

Unitermos: Maytenus truncata, Celastraceae, antiúlcera, cicatrizante, ratos.

\begin{abstract}
Evaluation of antiulcerogenic activity of Maytenus truncata Reiss (Celastraceae)". Maytenus truncata Reiss (Celastraceae) is a native plant from Bahia (Brazil), known as "espinheira-santa". It is popularly used in the form of decoct of leaves (tea) as antiulcerogenic, similarly to Maytenus ilicifolia, the true "espinheira-santa". This study aims to evaluate antiulcerogenic and healing activities, as well as the phytochemical profile, of ethyl acetate and methanol crude extracts of Maytenus truncata. Per os administration of these extracts at 120 $\mathrm{mg} / \mathrm{kg}$ and $240 \mathrm{mg} / \mathrm{kg}$ doses decreased the severity of gastric lesions induced by cold-restraint stress $\left(-18{ }^{\circ} \mathrm{C}\right.$ for 45 minutes) in rats, with more significant results for the crude methanol extract. The administration of the extracts caused $\mathrm{pH}$ increase. The results obtained with the administration of crude methanol extract are not contrary to its popular use, not only for the activity observed but, also, for its high polarity that enables the obtention of the active principles through infusion, though clinical studies should be performed to confirm this assertion.
\end{abstract}

Keywords: Maytenus truncata, Celastraceae, antiulcer, healing, rats.

\section{INTRODUÇ̃̃̃O}

Muitas espécies Maytenus são popularmente utilizadas na medicina tradicional brasileira, devido às suas atividades antiácida e antiulcerogênica (Cruz, 1982). Porém, o uso tradicional e a comercialização desse gênero têm precedido à avaliação farmacológica e toxicológica em estudos pré-clínicos (Souza-Formigoni et al., 1991; Gonzalez et al. 2001). A Maytenus truncata Reiss (Celastraceae) é uma planta nativa da Bahia (Brasil), sendo conhecida como "espinheira-santa". É usada popularmente na forma de decocto das folhas (chás) como antiulcerogênico, similarmente à Maytenus ilicifolia segundo Gonzalez et al. (2001), a verdadeira "espinheira-santa".

Souza-Formigoni (1991) comprovaram a ação protetora do abafado (infuso) de Maytenus ilicifolia contra o desenvolvimento da úlcera experimental em ratos, tanto por via oral como por via intraperitoneal. Os resultados obtidos nesse estudo, mostraram que o efeito protetor da "espinheira-santa" é comparado ao da cimetidina, a qual é utilizada como anti-histamínico de ação inibitória sobre a hemorragia digestiva e sobre a secreção gástrica de ácidos, comumente observados na úlcera péptica.

As propriedades antiulcerogênicas dessa espécie têm sido atribuídas aos triterpenos (Vilegas et al., 1994; Niero, 2001) e flavonóides (Vilegas et al., 1999; Leite et al., 2001). No entanto, Queiroga et al. (2000), avaliando 
o efeito antiúlcera do friedelan-3 $\beta$-ol e da friedelina (triterpenos), não observaram proteção gástrica.

O objetivo do presente estudo foi avaliar a eficácia antiulcerogênica de extratos em acetato de etila e metanol obtidos a partir do fracionamento do decocto das folhas de Maytenus truncata em lesões induzidas por estresse ao frio.

\section{MATERIAL E MÉTODOS}

\section{Material botânico}

As folhas de Maytenus ilicifolia (Celastraceae) foram coletadas no mês de julho de 2001 na cidade de Jequié, Bahia (exsicata $\mathrm{n}^{\circ}$ 25306A depositada no herbário do Museu de História Natural de Belo Horizonte, Minas Gerais), separadas, secas à temperatura ambiente e, em seguida, submetidas à moagem em moinho de facas. A extração foi realizada por decocção para obtenção de extrato aquoso semelhante ao material utilizado na medicina popular. $\mathrm{O}$ extrato aquoso foi preparado obedecendo-se a proporção de $10 \mathrm{~g}$ de folhas por litro de água. Aguardou-se o início da fervura, as folhas foram colocadas na água e, após três minutos, o material foi filtrado sob vácuo. A solução obtida foi submetida a aquecimento em temperatura de $50{ }^{\circ} \mathrm{C}$, sob ventilação, para evaporação da água, por 48 horas. O material obtido foi seco em dessecador, constituindo o decocto. O decocto, após pulverização em almofariz, foi submetido à extração exaustiva em aparelho de Soxhlet, utilizando, separadamente, acetato de etila e metanol. Cada extrato obtido, após remoção do solvente em evaporador rotatório, foi aquecido em banho de areia e guardado em dessecador para total remoção do solvente. $\mathrm{O}$ rendimento obtido foi de $1,2 \%$ e $6 \%$ para os extratos em acetato de etila e em metanol, respectivamente.

\section{Estudos fitoquímicos}

A prospecção química dos extratos em acetato de etila e metanol foi direcionada para polifenóis, taninos condensados, alcalóides, flavonóides, triterpenos e esteróides. Foram utilizados reagentes específicos adequados para cada classe de substâncias. Cada extrato foi submetido ao fracionamento em coluna cromatográfica utilizando sílica gel 60 , bem como cromatografia líquida de alta eficiência. Os constituintes químicos isolados foram caracterizados e identificados através de ponto de fusão, análise espectrométrica (infravermelho e ressonância magnética nuclear de hidrogênio e carbono 13) e comparação com amostra autêntica em cromatografia em camada delgada.

\section{Animais}

Ratos Wistar machos (220 $\pm 20 \mathrm{~g})$, provenientes do biotério da Faculdade de Farmácia da Universidade Federal de Minas Gerais (UFMG), foram usados. Cada grupo de experimento consistiu de 6 animais (3 por caixa), sendo mantidos em condições de temperatura $\left(23 \pm 2{ }^{\circ} \mathrm{C}\right)$ e umidade $(50 \%-60 \%)$ controladas e no ciclo claro-escuro de 12 h (07:00-19:00). Água e ração foram disponíveis ad libitum com exceção do dia que antecedeu os experimentos, sendo retirada a ração, $17 \mathrm{~h}$ anterior à administração das substâncias, mantendo-se livre acesso à água. O protocolo experimental ( $\left.\mathrm{n}^{\circ} 038 / 02\right)$ foi aprovado pelo Comitê de Ética em Experimentação Animal (CETEA) da UFMG.

\section{Avaliação da lesão gástrica em ratos}

Três modelos foram utilizados para avaliar a lesão gástrica, sendo os grupos testes tratados com os extratos e os controles com salina. No primeiro modelo, os extratos em acetato de etila $(120 \mathrm{mg} / \mathrm{kg}$ e $240 \mathrm{mg} / \mathrm{kg})$ e em metanol $(120 \mathrm{mg} / \mathrm{kg}$ e $240 \mathrm{mg} / \mathrm{kg})$, solubilizados em carboximetilcelulose $0,05 \%$, foram administrados ( 1 $\mathrm{ml} / 100 \mathrm{~g}$ de animal) per os (p.o.). Após $4 \mathrm{~h}$, os animais foram transferidos para um freezer $\left(-18{ }^{\circ} \mathrm{C}\right)$, em caixas individuais, e mantidos por $45 \mathrm{~min}$ de acordo com técnica modificada por Nascimento et al. (2003). Após esse período, os animais foram sacrificados em cuba saturada com vapores de éter e seus estômagos removidos.

No segundo modelo (antiúlcera), cimetidina $(100 \mathrm{mg} / \mathrm{kg}$ - SKF, Brasil) e extratos em acetato de etila $(120 \mathrm{mg} / \mathrm{kg}$ e $240 \mathrm{mg} / \mathrm{kg})$ e em metanol $(120 \mathrm{mg} / \mathrm{kg}$ e $240 \mathrm{mg} / \mathrm{kg}$ ) da M. truncata foram administrados p.o. por três dias consecutivos, uma vez ao dia, de acordo com técnica modificada descrita por Sairam et al. (2002). No quarto dia, os animais foram submetidos ao estresse ao frio, como descrito anteriormente. Após esse período, os animais foram sacrificados e seus estômagos removidos.

No terceiro modelo (cicatrização), os animais foram submetidos ao estresse ao frio no primeiro dia e tratados p.o. com cimetidina $(100 \mathrm{mg} / \mathrm{kg})$ e os extratos $(120 \mathrm{mg} / \mathrm{kg}$ e $240 \mathrm{mg} / \mathrm{kg}$ ) por três dias consecutivos. Seis horas após a última administração, os animais foram sacrificados e seus estômagos removidos.

Os estômagos foram abertos imediatamente após o sacrifício dos animais e lavados com $5 \mathrm{ml}$ de solução salina. Foram examinados em microscópio esterioscópio (Metrimpex-PZ, Labimex) e as lesões graduadas de acordo com sua severidade: $1+$ (petéquia $<1 \mathrm{~mm}$ ); 2 + (erosão de 1-3 mm) e $3+$ (erosão $>3 \mathrm{~mm})$. O índice de lesão (I.L.) foi calculado de acordo com Szelenyi e Thiemer (1978).

\section{pH gástrico}

Após remoção do estômago, uma pequena incisão foi feita próximo ao piloro e o $\mathrm{pH}$ gástrico foi medido, usando indicador de $\mathrm{pH}$ (Merck).

\section{Análise estatística}


Tabela 1. Efeito dos extratos em acetato de etila e em metanol da Maytenus truncata na severidade das lesões gástricas induzidas por estresse ao frio.

\begin{tabular}{|c|c|c|c|c|c|c|}
\hline Tratamento $(\mathrm{mg} / \mathrm{Kg})$ & \multicolumn{3}{|c|}{ Severidade da lesões gástricas } & I.L. total & \% Protecão & $\mathrm{pH}$ \\
\hline & L1 & L2 & L3 & & & \\
\hline Controle & $510,2 \pm 43,70$ & $381,8 \pm 30,10$ & $8 \pm 1,7$ & $216,3 \pm 25,9$ & - & 2 \\
\hline Metanol (120) & $294,6 \pm 30,84^{\mathrm{a}, \mathrm{b}}$ & $124,2 \pm 12,64^{\mathrm{a}, \mathrm{b}}$ & $1 \pm 1^{\mathrm{a}}$ & $91,0 \pm 8,6^{a, b}$ & 57,92 & $4^{\mathrm{a}}$ \\
\hline Acetato de Etila (120) & $512,4 \pm 55,84$ & $246,9 \pm 24,08^{\mathrm{a}}$ & $1,2 \pm 0,56^{\mathrm{a}}$ & $168,30 \pm 20,5$ & 22,19 & $4^{\mathrm{a}}$ \\
\hline Metanol (240) & $285,5 \pm 27,67^{\mathrm{a}, \mathrm{b}}$ & $114,0 \pm 13,17^{\mathrm{a}, \mathrm{b}}$ & $0.8 \pm 0,7^{\mathrm{a}}$ & $85,98 \pm 7,4^{\mathrm{a}, \mathrm{b}}$ & 60,24 & $4^{\mathrm{a}}$ \\
\hline Acetato de Etila (240) & $520,0 \pm 45,03$ & $235,8 \pm 27,1^{\mathrm{a}}$ & $1 \pm 1^{\mathrm{a}}$ & $165,77 \pm 18,3$ & 23,36 & $4^{\mathrm{a}}$ \\
\hline
\end{tabular}

Os valores representam média \pm E.P.M.

${ }^{a}$ Diferença significativa em relação ao grupo controle. $P<0,05(\mathrm{n}=6)$.

${ }^{\mathrm{b}}$ Diferença significativa em relação aos grupos tratados com o extrato em acetato de etila. $P<0,05(\mathrm{n}=6)$.

Tabela 2. Efeito antiúlcera dos extratos em acetato de etila e em metanol da Maytenus truncata e cimetidina sobre a mucosa gástrica no estresse induzido pelo frio.

\begin{tabular}{|c|c|c|c|c|c|c|}
\hline Tratamento $(\mathrm{mg} / \mathrm{Kg})$ & \multicolumn{3}{|c|}{ Severidade das lesões gástricas } & I.L. total & \% Proteção & $\mathrm{pH}$ \\
\hline & L1 & L2 & L3 & & & \\
\hline Controle & $415,7 \pm 22,7$ & $162,7 \pm 14,8$ & $10,7 \pm 2,2$ & $128,87 \pm 9,7$ & - & 2 \\
\hline Metanol (120) & $141,3 \pm 13,8^{\mathrm{a}, \mathrm{b}, \mathrm{c}}$ & $42,4 \pm 3,8^{a, b}$ & $1,1 \pm 0,5^{\mathrm{a}}$ & $38,23 \pm 5,9^{\mathrm{a}, \mathrm{b}, \mathrm{c}}$ & 70,33 & $4^{\mathrm{a}}$ \\
\hline Acetato de Etila (120) & $246,3 \pm 23,7^{\text {a }}$ & $67,8 \pm 4,9^{\mathrm{a}}$ & $2,1 \pm 0,91^{\mathrm{a}}$ & $64,50 \pm 5,4^{\mathrm{a}}$ & 49,79 & $4^{\mathrm{a}}$ \\
\hline Metanol (240) & $137,0 \pm 15,7^{\mathrm{a}, \mathrm{b}, \mathrm{c}}$ & $40,0 \pm 6,3^{\mathrm{a}, \mathrm{b}}$ & $1,1 \pm 1,2^{\mathrm{a}}$ & $36,72 \pm 3,1^{\mathrm{a}, \mathrm{b}, \mathrm{c}}$ & 71,50 & $4^{\mathrm{a}}$ \\
\hline Acetato de Etila (240) & $252,3 \pm 17,3^{\mathrm{a}}$ & $45,5 \pm 4,6^{\mathrm{a}}$ & $3,5 \pm 0,85^{\mathrm{a}}$ & $58,96 \pm 4,6^{\mathrm{a}}$ & 54,24 & $4^{\mathrm{a}}$ \\
\hline Cimetidina (100) & $92,5 \pm 6,4^{\mathrm{a}, \mathrm{d}}$ & $23,7 \pm 3,0^{\mathrm{a}, \mathrm{d}}$ & $1 \pm 1^{\mathrm{a}}$ & $23,73 \pm 5,5^{\mathrm{a}, \mathrm{d}}$ & 81,59 & $5^{\mathrm{a}}$ \\
\hline
\end{tabular}

Os valores representam média \pm E.P.M.

${ }^{a}$ Diferença significativa em relação ao grupo controle. $P<0,05(\mathrm{n}=6)$.

${ }^{\mathrm{b}}$ Diferença significativa em relação ao grupo tratado com o extrato em acetato de etila na dose de

$120 \mathrm{mg} / \mathrm{kg} . P<0,05(\mathrm{n}=6)$.

${ }^{c}$ Diferença significativa em relação ao grupo tratado com o extrato em acetato de etila na dose de

$240 \mathrm{mg} / \mathrm{kg} . P<0,05(\mathrm{n}=6)$.

${ }^{\mathrm{d}}$ Diferença significativa em relação aos grupos tratados com os extratos em acetato de etila e em metanol. $P<0,05(\mathrm{n}=6)$.

Tabela 3. Efeito cicatrizante dos extratos em acetato de etila e em metanol da Maytenus truncata e cimetidina sobre a mucosa gástrica no estresse induzido pelo frio.

\begin{tabular}{|c|c|c|c|c|c|c|}
\hline Tratamento $(\mathrm{mg} / \mathrm{Kg})$ & \multicolumn{3}{|c|}{ Lesões gástricas } & I.L. total & \% Proteção & $\mathrm{pH}$ \\
\hline & L1 & L2 & L3 & & & \\
\hline Controle & $400,8 \pm 35,5$ & $167,0 \pm 24,7$ & $14,3 \pm 3,7$ & $129,62 \pm 17,7$ & - & 2 \\
\hline Metanol (120) & $147,8 \pm 16,3^{\mathrm{a}, \mathrm{b}}$ & $34,3 \pm 3,2^{\mathrm{a}}$ & $0,5 \pm 0,4^{\mathrm{a}}$ & $36,32 \pm 4,31^{a, b}$ & 71,97 & $4^{\mathrm{a}}$ \\
\hline Acetato de Etila (120) & $244,9 \pm 25,6^{\mathrm{a}}$ & $44,1 \pm 5,8^{\mathrm{a}}$ & $0,6 \pm 0,3^{a}$ & $55,82 \pm 5,7^{\mathrm{a}}$ & 56,93 & $4^{\mathrm{a}}$ \\
\hline Metanol (240) & $134,0 \pm 12,2^{\mathrm{a}, \mathrm{b}}$ & $30,0 \pm 6,5^{\mathrm{a}}$ & $1 \pm 0,5^{\mathrm{a}}$ & $32,83 \pm 4,1^{\mathrm{a}, \mathrm{b}}$ & 74,67 & $4^{\mathrm{a}}$ \\
\hline Acetato de Etila (240) & $235,8 \pm 12,4^{\mathrm{a}}$ & $40,5 \pm 8,6^{\mathrm{a}}$ & $1,5 \pm 0,5^{\mathrm{a}}$ & $53,55 \pm 3,9^{a}$ & 58,69 & $4^{\mathrm{a}}$ \\
\hline Cimetidina (100) & $80,8 \pm 10,8^{\mathrm{a}, \mathrm{c}}$ & $30,2 \pm 6,3^{\mathrm{a}}$ & $0,5 \pm 0,4^{\mathrm{a}}$ & $23,78 \pm 4,8^{\mathrm{a}, \mathrm{b}}$ & 81,65 & $5^{\mathrm{a}}$ \\
\hline
\end{tabular}

Os valores representam média \pm E.P.M.

${ }^{a}$ Diferença significativa em relação ao grupo controle. $P<0,05(\mathrm{n}=6)$.

${ }^{\mathrm{b}}$ Diferença significativa em relação aos grupos tratados com o extrato em acetato de etila.P $<0,05(\mathrm{n}=6)$.

${ }^{c}$ Diferença significativa em relação aos grupos tratados com os extratos em acetato de etila e em metanol.

$P<0,05(\mathrm{n}=6)$.

Método ANOVA one way, seguido por múltipla comparação (Correção por Bonferroni), foi utilizado para analisar os resultados. A significância estatística considerada foi $p<0,05$ (limite de confiança: $95 \%$ ).

\section{RESULTADOS}

\section{Estudos fitoquímicos}

$\mathrm{Na}$ avaliação fitoquímica do extrato em acetato de etila verificou-se a presença de alcalóides, bem como de compostos de natureza flavonoídica como quercetina, 4'-O-metil-epigalocatequina e proantocianidina $\mathrm{A}$, enquanto para o extrato metanólico foi detectado apenas 
o flavonóide, proantocianidina A.

\section{Avaliação das lesões gástricas em ratos}

A severidade das lesões $1+, 2+\mathrm{e} 3+$ nos animais tratados com os extratos em acetato de etila e metanol $(120 \mathrm{mg} / \mathrm{kg}$ e $240 \mathrm{mg} / \mathrm{kg})$ de M. truncata são apresentados na Tabela 1 . O extrato em metanol apresentou diferença significativa em todos os níveis em relação ao controle, e nos níveis $1+$ e $2+$ em relação ao acetato de etila. Diferença significativa entre o extrato em acetato de etila e controle foi observada nos níveis $2+$ e $3+$.

Como apresentado na Tabela 2, o número de lesões na mucosa gástrica em animais tratados com os extratos em acetato de etila e em metanol $(120 \mathrm{mg} /$ $\mathrm{kg}$ e $240 \mathrm{mg} / \mathrm{kg}$ ) de $M$. truncata mostraram valores estatisticamente menores que o grupo controle. $\mathrm{O}$ extrato metanólico apresentou resultados mais significativos em relação ao extrato em acetato de etila nos níveis $1+$ e 2+, na dose de $120 \mathrm{mg} / \mathrm{kg}$ e no nível 1+ na de $240 \mathrm{mg} / \mathrm{kg}$. Diferença significativa entre cimetidina e os extratos foi observada nos níveis $1+$ e $2+$.

$\mathrm{O}$ efeito cicatrizante (Tabela 3 ) dos extratos em acetato de etila e em metanol $(120 \mathrm{mg} / \mathrm{kg}$ e $240 \mathrm{mg} / \mathrm{kg})$ apresentou diferença significativa em relação à cimetidina $(100 \mathrm{mg} / \mathrm{kg})$ somente no nível 1+. A severidade das lesões nos animais tratados com os extratos e cimetidina foram significativamente menores quando comparadas ao grupo controle em todos os níveis.

\section{Efeito dos extratos sobre o $\mathbf{p H}$}

Conforme apresentado nas Tabelas 1, 2 e 3, foi observado aumento significativo do $\mathrm{pH}$ tanto na administração dos extratos em acetato de etila e em metanol ( $120 \mathrm{mg} / \mathrm{kg}$ e $240 \mathrm{mg} / \mathrm{kg}$ ) quanto na da cimetidina $(100 \mathrm{mg} / \mathrm{kg})$ em relação ao grupo controle.

\section{DISCUSSÃO}

O perfil fitoquímico da $M$. truncata revelou a predominância dos flavonóides quercetina, 4'-Ometil-epigalocatequina e proantocianidina $\mathrm{A}$ no extrato em acetato de etila e de proantocianidina A no extrato metanólico. A ausência de triterpenos e esteróides nos extratos em acetato de etila e em metanol pode ser atribuída à baixa polaridade desses grupos de substâncias.

A infusão de folhas da M. ilicifolia tem sido amplamente utilizada na medicina tradicional brasileira no tratamento de dispepsia, gastrite crônica e úlcera gástrica (Souza-Formigoni et al., 1991). Segundo Lewis e Hanson (1991) os triterpenos, compostos isolados da Maytenus spp. (Itokawa et al., 1991; Vilegas et al., 1994; Niero et al., 2001), são, provavelmente, os responsáveis pela atividade antiulcerogênica.

No entanto, Vilegas et al. (1999) e Leite et al. (2001) verificaram atividade antiulcerogênica de flavonóides tetrasacarídeos obtidos a partir da $M$. aquifolium. Segundo Gonzalez e Di Stasi (2002) os flavonóides podem ser reconhecidos como compostos ativos contra lesões gástricas devido o aumento de fatores de proteção ou atividade antioxidante.

No presente estudo, as lesões gástricas em animais submetidos ao estresse ao frio diminuiu significativamente, em relação ao controle, quando os mesmos foram tratados com os extratos em acetato de etila, lesões $1+$ e 2+, e em metanol, lesões 1+, 2+ e 3+, nas doses utilizadas. $\mathrm{O}$ extrato em metanol apresentou melhores resultados de proteção gástrica no índice total, $57,92 \%$ e $60,24 \%$ nas doses de $120 \mathrm{mg} / \mathrm{kg}$ e $240 \mathrm{mg} / \mathrm{kg}$, respectivamente, contra $22,19 \%$ e $23,36 \%$ do extrato em acetato de etila. No entanto, não houve diferença entre os extratos na lesão 3+ (hemorrágicas) entre ambos. Sendo essa lesão a mais grave, justifica-se a avaliação antiúlcera e cicatrizante do extrato em acetato de etila, além do metanólico. Nossos resultados estão de acordo com aqueles obtidos por Aguwa e Lawal (1988) que também observaram atividade antiulcerogênica de flavonóides, utilizando o modelo de estresse ao frio.

Úlceras provocadas por estresse são devido a fatores psicológicos e fisiológicos (Miller, 1987). O estresse induz a peroxidação lipídica a partir do aumento dos níveis de peroxidase lipídica. A conseqüência desse processo é o aumento da geração de espécies livres de oxigênio reativo ocasionando, conseqüentemente, dano oxidativo que é considerado fator comum na patogenia de diferentes modelos experimentais e clínicos de úlcera (Goel; Bhattacharya, 1991; Sairam et al., 2002).

Mais de 4000 flavonóides têm sido descritos e classificados em categorias como flavonóis, flavonas, catequinas, flavononas, antocianidinas e isoflavonas, com grande variedade de efeitos biológicos, como anti-neoplásico, antiinflamatório, hepatoprotetor, antihipertensivo e anti-plaquetário, tanto in vitro quanto in vivo (Formica; Regelson, 1995; Hollman; Batan, 1997). Além desses, o efeito antioxidante tem sido observado também em alguns estudos (Oshima et al., 1998; Magnani et al., 2000). Mora et al. (1990) e Cotelle et al. (1992), além da atividade antioxidante, verificaram ainda que os flavonóides atuam sobre radicais hidroxilas, devido sua capacidade de doar hidrogênio.

No presente estudo, os extratos utilizados apresentaram resultados mais significativos nos efeitos antiúlcera e cicatrizante quando comparados com o grupo controle. No efeito antiúlcera, diferença significativa foi observada entre o grupo tratado com extrato em acetato de etila tanto na dose de $120 \mathrm{mg} / \mathrm{kg}$, que foi de $47,60 \%$, quanto na dose de $240 \mathrm{mg} / \mathrm{kg}$, que foi de $57,22 \%$, em relação ao extrato em metanol, que foi de 70,33\% (120 $\mathrm{mg} / \mathrm{kg})$ e $71,50 \%(240 \mathrm{mg} / \mathrm{kg})$, e cimetidina que foi de $81,59 \%(100 \mathrm{mg} / \mathrm{kg})$.

No efeito cicatrizante, também foi observada diferença significativa entre o grupo tratado com acetato de etila na dose de $120 \mathrm{mg} / \mathrm{kg}$, que foi de $56,93 \%$, e na 
dose de $240 \mathrm{mg} / \mathrm{kg}$, que foi de $58,69 \%$, em relação ao extrato em metanol, que foi de $71,97 \%(120 \mathrm{mg} / \mathrm{kg})$ e $74,67 \%(240 \mathrm{mg} / \mathrm{kg})$, e cimetidina que foi de $81,65 \%$ $(100 \mathrm{mg} / \mathrm{kg})$.

Conforme mostram os resultados, tanto na avaliação antiúlcera quanto na de cicatrização, o extrato em metanol, além de apresentar melhor proteção gástrica, quando comparado ao extrato em acetato de etila, não diferiu significativamente em relação ao grupo tratado com cimetidina. Além disso, o extrato em metanol nas doses de $120 \mathrm{mg} / \mathrm{kg}$ e $240 \mathrm{mg} / \mathrm{kg}$, no modelo experimental empregado, apresentou atividade gastroprotetora similar indicando o alcance de um platô. No extrato em acetato de etila também foi observado esse fenômeno, com exceção da lesão $2+$ do efeito antiúlcera. Porém, não foi observada também diferença significativa na atividade gastroprotetora na administração de ambas as doses.

$O$ fato de o extrato em acetato de etila não apresentar efeito antiulcerogênico significativo, apesar da presença dos flavonóides identificados, deve-se, provavelmente, à necessidade do uso de doses maiores ou tempo maior de administração, pois, como pode ser observado houve diminuição significativa das lesões $1+$ e $2+$ nos experimentos de múltiplas doses (antiúlcera e cicatrizante) em relação ao de dose única.

$\mathrm{Na}$ avaliação antiúlcera e cicatrização, foi verificado, também, aumento significativo no $\mathrm{pH}$ gástrico em relação ao grupo controle. Porém, não houve diferença significativa entre o $\mathrm{pH}$ gástrico dos grupos tratados com os extratos e cimetidina.

Esses resultados estão de acordo com aqueles obtidos em estudos envolvendo a espécie Maytenus. SouzaFormigoni et al. (1991) e Antônio e Souza-Brito (1998) avaliando o potencial antiulcerogênico da $M$. ilicifolia e da $M$. aquifolium, respectivamente, verificaram que as mesmas protegem a mucosa gástrica, quando comparadas com a cimetidina. Nesses estudos foi observado também que a proteção gástrica é acompanhada pelo aumento do $\mathrm{pH}$. Nossos resultados estão de acordo também com aqueles obtidos por Gonzalez e Di Stasi (2002) que concluíram que os flavonóides podem ser reconhecidos como compostos ativos contra lesões gástricas .

Assim, os dados obtidos na administração do extrato em metanol da $M$. truncata não contrariam seu uso popular, não somente pela atividade observada, mas também por se tratar de um extrato de alta polaridade, cujos princípios podem ser obtidos a partir de uma infusão, embora estudos clínicos devam ser realizados para confirmação dessa hipótese. Concluindo, os resultados tornam a planta interessante alvo de estudo, visando o desenvolvimento de fitomedicamentos ou a busca de novas entidades químicas com ação antiulcerogênica.

\section{REFERENCIAS}

Aguwa CN, Lawal AM 1988. Pharmacological studies on the active principles of Calliandra portoricensis leaf extracts. J Ethnopharmacol 22: 63-71.

Antônio MA, Souza-Brito ARM 1998. Oral anti-inflammatory and anti-ulcerogenic activities of a hydroalcoholic extract and partitioned fractions of Turnera ulmifolia (Turneraceae). J Ethnopharmacol 61: 215-228.

Cotelle N, Bernier JL, Henichart JP, Catteau JP, Gaydou F, Wallet JC 1992. Scavenger and antioxidant properties of tem synthetic flavones. Free Radical Bio Med 12: 211-219.

Cruz GL 1982. Dicionário das plantas úteis do Brasil. São Paulo: Ed. Civilização Brasileira.

Formica JV, Regelson W 1995. Review of the biology of quercetin and related bioflavonoids. Food Chem Toxicol 33: 1061-1080.

Goel RK, Bhattacharya SK 1991. Gastroduodenal mucosal defense and mucosal protective agents. Indian J Exp Biol 29: 701-714.

Gonzalez FG, Portela TY, Stipp EJ, Di Stasi LC 2001. Antiulcerogenic and analgesic effects of Maytenus aquifolium, Sorocea bomplandii and Zolernia ilicifolia. J Ethnopharmacol 77: 41-47.

Gonzalez FG, Di Stasi LC 2002. Antiulcerogenic and analgesic activities of the leaves of Wilbrandia ebracteata in mice. Phytomedicine 9: 125-134.

Hollman PCH, Batan MB 1997. Absorption, metabolism and health effects of dietary flavonoids in man. Biomed Pharmacother 51: 305-310.

Itokawa H, Shirota O, Ikuta H, Morita H, Takeya K, Itaka Y 1991. Triterpenes from Maytenus ilicifolia. Phytochemistry 30: 3713-3716.

Leite JPV, Rastrelli L, Romussi G, Oliveira AB, Vilegas JHY, Vilegas W, Pizza C 2001. Isolation and HPLC quantitative analysis of flavonoid glycosids from Brazilian beverages (Maytenus ilicifolia and Maytenus aquifolium). J Agr Food Chem 49: 3796-3801.

Lewis DA, Hanson PJ 1991. Anti-ulcer drugs of plant origin. In: Ellis GP (Eds.) Progress Medicinal Chemistry. New York: Elsevier Science Publishers vol. 28, p. 201-231.

Magnani L, Gaydou EM, Hubaud JC 2000. Spectrophotometric measurement of antioxidant properties of flavones and flavonols against superoxide anion. Anal Chim Acta 411: 209-216.

Miller TA 1987. Mechanism of stress-related mucosal damage. Am J Med 83: 8-14.

Mora A, Payá M, Rios JL, Alcaraz MJ 1990. Structureactivity relationships of polymetoxyflavones and other flavonoids as inhibitors of non enzymic lipid peroxidation. Biochem Pharmacol 40: 793-797.

Nascimento JWL, Santos LH, Nothenberg MS, Coelho MM, Oga S, Tagliati CA 2003. Anti-inflammmatory activity and gastric lesions induced by zinc-tenoxicam. Pharmacology 68: 64-69.

Niero R, Moser R, Busato ACB, Yunes RA, Reis A 2001. A comparative chemical study of Maytenus ilicifolia Mart. Reiss and Maytenus robusta. Reiss (Celastraceae). Z Naturforsch C 56: 158-161.

Oshima H, Yoshie Y, Auriol S, Gilbert I 1998. Antioxidant and pro-oxidant actions of flavonoids: effects on DNA damage induced by nitric oxide, peroxynitrile and nitroxyl anion. Free Radical Bio Med 25: 1057-1065.

Queiroga CL, Silva GF, Dias PC, Possenti A, Carvalho JE 2000. Evaluation of the antiulcerogenic activity of friedelan- 
$3 \beta$-ol and friedelin isolated from Maytenus ilicifolia (Celastraceae). J Ethnopharmacol 72: 465-468.

Sairam K, Rao CHV, Dora Babu M, Vijay Kumar K, Agrawal VK, Goel RK 2002. Antiulcerogenic effect of methanolic extract of Emblica officinallis: an experimental study. J Ethnopharmacol 82: 1-9.

Souza-Formigoni MLO, Oliveira MGM, Monteiro MG, SilveiraFilho NG, Braz S, Carlini EA 1991. Antiulcerogenic effects of two Maytenus species in laboratory animals. J Ethnopharmacol 34: 21-27.

Szelenyi I, Thiemer K 1978. Distention ulcer as a model for testing of drugs for ulcerogenic side effects. Arch Toxicol 41: 99-105.

Vilegas JHI, Lanças FM, Cervi AC 1994. High-resolution gas-chromatography analysis of 'espinheira-santa' (Maytenus ilicifolia and Maytenus aquifolium): analysis of crude drug adulterations. Phytother Res 8 : 241-244.

Vilegas W, Sanommiya M, Rastrelli L, Pizza C 1999. Isolation and structure elucidation of two new flavonoid glycosides from the infusion of Maytenus aquifolium leaves. Evaluation of the antiulcer activity of the infusion. J Agr Food Chem 47: 403-406. 